\title{
Evidence of microglial activation in autism and its possible role in brain underconnectivity
}

\author{
JUAN I. RODRIGUEZ ${ }^{1}$ AND JANET K. KERN ${ }^{1,2,3}$
}

\begin{abstract}
Evidence indicates that children with autism spectrum disorder (ASD) suffer from an ongoing neuroinflammatory process in different regions of the brain involving microglial activation. When microglia remain activated for an extended period, the production of mediators is sustained longer than usual and this increase in mediators contributes to loss of synaptic connections and neuronal cell death. Microglial activation can then result in a loss of connections or underconnectivity. Underconnectivity is reported in many studies in autism. One way to control neuroinflammation is to reduce or inhibit microglial activation. It is plausible that by reducing brain inflammation and microglial activation, the neurodestructive effects of chronic inflammation could be reduced and allow for improved developmental outcomes. Future studies that examine treatments that may reduce microglial activation and neuroinflammation, and ultimately help to mitigate symptoms in ASD, are warranted.
\end{abstract}

Keywords: Autism, autism spectrum disorder (ASD), microglial activation, neuroinflammation, underconnectivity, early intervention, treatments

\section{EVIDENCE DF MICRDGLIAL ACTIVATION AND BRAIN INFLAMMATIONIN AUTISM}

A study by Johns Hopkins University School of Medicine found evidence of microglial activation in individuals with autism or autism spectrum disorder (ASD) (Pardo et al., 2005). Indeed, several studies now provide evidence that children with autism suffer from an ongoing neuroinflammatory process in different regions of the brain involving microglial activation (Enstrom et al., 2005; Pardo et al., 2005; Vargas et al., 2005; Zimmerman et al., 2005). Evidence of neuroinflammation includes activated microglia and astrocytes found in post-mortem brain tissue (Pardo et al., 2005; Vargas et al., 2005; Morgan et al., 2010) and irregular, proinflammatory cytokine profiles in the brain and cerebrospinal fluid of children with ASD (Vargas et al., 2005; Zimmerman et al., 2005; Chez et al., 2007). Neuroinflammation, in general, is characterized by the reactivity of microglial cells and astrocytes, activation of inducible nitric oxide (NO)-synthase (i-NOS) and increased expression and/or release of cytokines and chemokines (Monnet-Tschudi et al., 2011) and that is what is found in autism. As Herbert (2005) pointed out in her review of the brain abnormalities in ASD, the autistic brain is not simply wired differently, but neuroinflammation is a part of the pathology in autism from childhood through adulthood.

For example, three post-mortem studies have shown microglial activation in ASD. First, Vargas et al. (2005)

Corresponding author:

Janet K. Kern

Email: jkern@dfwair.net examined brain tissue and cerebral spinal fluid (CSF) in those with autism. For the morphological studies, brain tissues from the cerebellum, midfrontal and cingulate gyrus were obtained at autopsy from 11 patients with autism. Fresh-frozen tissues from seven patients and CSF from six living patients with autism were used for cytokine protein profiling. The authors found active neuroinflammatory process in the cerebral cortex, white matter, and notably in cerebellum of patients with autism, with marked activation of microglia and astroglia. The authors stated that the CSF showed a unique proinflammatory profile of cytokines. The authors also stated that the pattern of cellular and protein findings suggests that the brain's own immune system (not immune abnormalities from outside the brain) and the neuroinflammatory process appears to be an ongoing and chronic mechanism of central nervous system (CNS) dysfunction.

Second, Morgan et al. (2010) examined the dorsolateral prefrontal cortex of male cases with autism $(n=13)$ and control cases $(n=9)$ and found microglial activation and increased microglial density in the dorsolateral prefrontal cortex in those with autism. They also noted process retraction and thickening, and extension of filopodia (small protrusions sent out from a migrating cell in the direction that it wants to move) from the processes. The authors stated that the microglia were markedly activated in 5 of 13 cases with autism, including 2 of 3 under age 6, and marginally activated in an additional 4 of 13 cases. The authors stated that because of its early presence, microglial activation may play a central role in the brain pathogenesis of autism.

Third, Tetreault et al. (2012) immunocytochemically identified microglia in fronto-insular and visual cortex in autopsy brains of well-phenotyped subjects with autism and matched controls and stereologically quantified the microglial densities. They found that in the fronto-insular and visual cortex, 
individuals with autism had significantly more microglia compared to controls. The authors concluded that because they observed increased densities of microglia in two functionally and anatomically disparate cortical areas, microglia are probably denser throughout cerebral cortex in brains of people with autism.

\section{EVIDENCE DF ASTRDCYTIC ACTIVATION IN THE BRAIN IN A UTISM}

Numerous studies have shown that glial fibrillary acidic protein (GFAP) levels are increased in autism. An autopsy report by Bailey et al. (1998), for example, found that the Purkinje cell loss in ASD was sometimes accompanied by gliosis and an increase in GFAP.

A study by Ahlsen et al. (1993) examined the levels of GFAP in the CSF of children with autism and found that their average GFAP level was three times higher than it was in the control group. The authors stated that the results could implicate gliosis and unspecified brain damage in children with autism. Laurence and Fatemi (2005) examined levels of GFAP in the frontal, parietal and cerebellar cortices using age-matched autistic and control post-mortem specimens. GFAP was significantly elevated in all three brain areas. The authors stated that the elevated GFAP confirms microglial and astroglial activation in autism and indicates gliosis, reactive injury, and perturbed neuronal migration processes.

Rosengren et al. (1992) also found GFAP levels in CSF in children with autism were higher than those in normal control children of the same age range. The authors stated that the high levels of GFAP in combination with normal $\mathrm{S}-100$ protein concentrations in CSF indicate reactive astrogliosis in the CNS.

Also, Fatemi et al. (2008) investigated whether two astrocytic markers, aquaporin 4 and connexin 43, are altered in Brodmann's Area 40 (BA40, parietal cortex), BA9 (superior frontal cortex) and the cerebella of brains of subjects with autism and matched controls. The authors reported that the findings demonstrated significant changes in the two astrocytic markers in the brain from individuals with autism.

\section{RESULTS DF EXTENDED MICRDGLIAL AND ASTRDCYTIC ACTIVATION}

When microglia remain activated for an extended period, the production of mediators is sustained longer than usual. This increase in mediators contributes to loss of synaptic connections and neuronal death (Wood, 2003). Streit et al. (2004) state that in the case of chronic neuroinflammation, the cumulative ill effects of microglial and astrocytic activation can contribute to and expand the initial neurodestruction, thus maintaining and worsening the disease process through their actions. Neuroinflammation generally refers to more chronic, sustained injury when the responses of microglial cells expand the neurodestructive effects (Streita, 2006). Evidence suggests that the collateral neural damage can involve loss of connections in the brain (Gehrmann et al.,
1995). Underconnectivity is found in autism and will be discussed in the next section.

\section{EVIDENCE DF ABNDRMAL BRAIN CONNECTIVITYINASD}

It is apparent from many studies that ASD involves the loss of critically important neuronal connections and networks (Just et al., 2007; Kana et al., 2009; Minshew and Keller, 2010; Di Martino et al., 2011; Wass, 2011). In a recent review of connectivity in ASD, Wass (2011) stated that there is 'considerable convergent evidence suggesting that connectivity is disrupted in ASD'. From his review of the literature, he states that the evidence indicates long-distance underconnectivity, and that disruptions appear more severe in the later-developing cortical regions.

As a result, the functional connectivity among regions of autistic brains is diminished (Herbert et al., 2004, 2005; Herbert, 2005). For example, Damarla et al. (2010) investigated the cortical underconnectivity theory in autism by examining the neural bases of the visuospatial processing in high-functioning autism. Using a combination of behavioral, functional magnetic resonance imaging (fMRI), functional connectivity and corpus callosum morphometric methodological tools, they found that the autism group had lower functional connectivity between the higher-order working memory/executive areas and the visuospatial regions (between frontal and parietal-occipital).

Ebisch et al. (2011), using fMRI, found reduced functional connectivity in ASD, compared with controls, between anterior and posterior insula and specific brain regions involved in emotional and sensory processing. Di Martino et al. (2011) found that children with ASD have abnormal functional connectivity between nearly all striatal subregions and heteromodal associative and limbic cortex previously implicated in the physiopathology of ASD (e.g. insular and right superior temporal gyrus).

Shukla et al. (2010) found fiber tract abnormalities in the corpus callosum (indicating impaired interhemispheric transfer), internal capsule and middle cerebellar peduncle and all three segments of the internal capsule in ASD. Boger-Megiddo et al. (2006) assessed midsagittal corpus callosum cross-sectional areas in 3-4 year olds with ASD compared to typically developing (TD) and developmentally delayed (DD) children. Although there was no difference in absolute size compared to TD, ASD callosums were disproportionately small when adjusted for increased ASD cerebral volume. The ASD clinical subgroup analysis revealed greater proportional callosum reduction in the more severely affected autistic disorder than in pervasive developmental disorder-not otherwise specified children. Just et al. (2007) found that relevant parts of the corpus callosum, through which many of the bilaterally activated cortical areas communicate, were smaller in cross-sectional area in the autistic participants and that the size of the genu of the corpus callosum was correlated with frontal-parietal functional connectivity.

Particularly implicated in connectivity is the cerebellum, one of the most common sites of anatomic abnormality in autism (Courchesne, 1997; Courchesne and Pierce, 2002; Belmonte et al., 2004). The Purkinje cell is the main output cell in the cerebellum and it is significantly diminished in number in ASD (Palmen et al., 2004). 
It is important to note that the connectivity issues are related to the symptomatology in ASD. Using electroencephalography (EEG) to assess dynamic brain connectivity, Barttfeld et al. (2011), for example, found that the greater the abnormalities found in the connectivity in ASD, the worse the child's symptoms.

\section{EVIDENCE DF INCREASED PRDINFLAMMATDRY CYTOKINE LEVELS IN THE BRAIN AND CSF IN $A \cup T I S M(T N F-\alpha, I F N-\gamma, I L-1 \beta, I L-B)$}

As mentioned earlier, there is evidence of proinflammatory cytokine profiles in the brain and cerebrospinal fluid of children with ASD (Vargas et al., 2005; Zimmerman et al., 2005; Chez et al., 2007). Some specific examples are as follows. Li et al. (2009) showed that proinflammatory cytokines (tumor necrosis factor- $\alpha$ (TNF- $\alpha$ ), interleukin (IL)-6 and granulocyte-macrophage colony-stimulating factor (GM-CSF)), Th1 cytokine (IFN- $\gamma$ ) and chemokine (IL-8) were significantly increased in the brains of ASD patients compared with the controls. A study by Vargas et al. (2005) demonstrated tumor growth factor- $\beta_{1}$, derived from neuroglia, was significantly increased in the middle frontal gurus of autistic patients, while macrophage chemoattractant protein (MCP)-1, IL-6 and IL-10 were increased in the anterior cingulated gurus. In addition, using protein array approach, Vargas and colleagues also found that MCP-1, IL-6, IL-8, and IFN- $\gamma$ were significantly increased in the CSF (Vargas et al., 2005). TNF- $\alpha$ was also shown to be increased in the CSF of autistic patients by Chez et al. (2007). Chez et al. stated that the elevation of cerebrospinal fluid levels of TNF- $\alpha$ was significantly higher (mean $=$ 104.10 $\mathrm{pg} \mathrm{ml}^{-1}$ ) than concurrent serum levels (mean $=2.78$ $\mathrm{pg} \mathrm{ml}^{-1}$ ) in all the patients studied. They stated that the ratio was significantly higher than the elevations reported for other pathological states for which cerebrospinal fluid and serum TNF- $\alpha$ levels have been simultaneously measured and that this finding may provide an insight into CNS inflammatory mechanisms in autism.

\section{PDSSIBLERDLE DF NF-кB EXPRESSION IN NEUROINFLAMMATION IN AUTISM}

The neuroinflammation in autism appears to be chronic and even excessive. Recent research suggests that the exaggerated brain immune response in ASD may be due, in part, to aberrant nuclear factor kappa-light-chain enhancer of activated $B$ cells (NF- $\kappa \mathrm{B}$ or NF-kappaB) expression, which can produce chronic or excessive inflammation (Young et al., 2011). A recent study by Young et al. (2011) examined NF- $\mathrm{KB}$ in human post-mortem samples of orbitofrontal cortex tissue in autism as compared to controls. They hypothesized that the concentrations of NF- $\mathrm{KB}$ would be elevated, especially in activated microglia in ASD, and $\mathrm{pH}$ would be concomitantly reduced (i.e. acidification). According to the authors, neurons, astrocytes, and microglia all demonstrated increased extranuclear and nuclear translocated NF- $\kappa$ B p65 expression in brain tissue from ASD donors relative to samples from matched controls. The between-group differences were increased in astrocytes and microglia relative to neurons, but particularly pronounced for highly 'mature microglia'. Measurement of $\mathrm{pH}$ in homogenized samples demonstrated a 0.98-unit difference in means and a strong $(F=98.3 ; P=0.00018)$ linear relationship to the expression of nuclear translocated NF- $\kappa B$ in mature microglia. Young et al. (2011) summarized that NF- $\mathrm{kB}$ is aberrantly expressed in orbitofrontal cortex in patients with ASD, as part of a putative molecular cascade leading to inflammation, especially of resident immune cells in brain regions associated with the behavioral and clinical symptoms of ASD. Their study provides further evidence of neuroinflammation that may be categorized as excessive in ASD.

Naik et al. (2011) examined NF- $\mathrm{B}$ in peripheral blood samples of 67 children with autism and 29 control children using electrophoretic mobility shift assay. They stated that there was a significant increase in NF- $\kappa$ B DNA binding activity in peripheral blood samples of children with autism and when the fold increase of NF-кB in cases $(n=67)$ was compared with that of controls $(n=29)$, there was a significant difference (3.14 versus 1.40, respectively; $P<0.02)$. They concluded that autism may arise, at least in part, from an NF- $\mathrm{B}$ pathway gone awry.

Evidence suggests that the equivalent of a vicious cycle can occur where microglia produce oxidative products and then increased intracellular reactive oxygen species (ROS), in turn, activates a redox-sensitive NF- $\mathrm{KB}$ to provoke excessive neuroinflammation. According to Nakanishi et al. (2011), this can result in memory deficits and prolonged behavioral consequences.

\section{POSSIBLE ROLE DF GLUTATHIONE DEPLETION IN MICRDGLIAL ACTIVATIONIN AUTISM}

Recent research suggests that glutathione (GSH) depletion can play a role in microglia-mediated neurotoxicity (Lee et al., 2010). Lee et al. (2010), for example, explored whether GSH depletion stimulated a neuroinflammatory response. They found that inhibition of GSH biosynthesis with D,L-buthionine-S, $R$-sulfoximine causes human microglia and human astrocytes to release TNF- $\alpha$, IL- 6 and nitrite ions. They concluded that as astrocytes are a main supplier of GSH to microglia and neurons in the brain, depletion of GSH during aging or neurodegeneration in neurological diseases may not only lead to activation of microglia and the astrocytes themselves, but may also render neurons sensitive to cell death.

In addition, Kigerl et al. (2011) found that ex vivo analyses showed that redox balance in microglia and macrophages is controlled by induction of glutamate/cystine antiporter (system $\mathrm{x}(\mathrm{c})(-))$ and that high GSH:GSSG ratios predict the neurotoxic potential of these cells. [Reduced glutathione (GSH) is a major tissue antioxidant. The formation of a disulfide bond between two GSH molecules gives rise to oxidized glutathione (GSSG). Under conditions of oxidative stress, GSSG accumulates and the ratio of GSH to GSSG will decrease. Therefore, the GSH/GSSG ratio can be used as an indicator of oxidative stress in cells and tissues.]

More importantly, studies suggest that children with ASD have inadequate GSH production. First, studies indicate abnormalities in the transsulfuration pathway in ASD (the pathway is where GSH is made) (James et al., 2004, 2006; Geier et al., 2009). And studies show low plasma GSH levels 
in ASD (James et al., 2004, 2006; Geier et al., 2009) and reduced glutathione regenerating enzymes (Al-Yafee et al., 2011). In addition, James et al. (2009) used lymphoblastoid cells (LCLs) derived from autistic children and unaffected controls to assess relative concentrations of reduced glutathione (GSH) and oxidized disulfide glutathione (GSSG) in cell extracts and isolated mitochondria as a measure of intracellular redox capacity. Their results indicated that the GSH/ GSSG redox ratio decreased and the percentage of oxidized glutathione increased in both cytosol and mitochondria in the autism LCLs.

More importantly, Chauhan et al. (2012) compared DNA oxidation and glutathione redox status in post-mortem brain samples from the cerebellum and frontal, temporal, parietal and occipital cortex from autistic subjects and agematched normal subjects. The authors reported that levels of reduced glutathione GSH were significantly reduced and the levels of oxidized glutathione GSSG were significantly increased in the cerebellum and temporal cortex in the brain samples from the group with autism as compared to the corresponding levels in the control brain samples.

Thus, it is possible that inadequate availability of GSH in ASD may play a role in microglial activation. Furthermore, GSH stores could be depleted from oxidative stress caused by microglial activation and if an individual cannot readily regenerate GSH, low GSH availability may stimulate microglial activation, leading to a cascade of events that potentiates itself.

In addition, in its resting state, microglia have been shown to contain levels of glutathione significantly higher than in astrocytes or neurons (Chatterjee et al., 2000; Hirrlinger et al., 2000). It appears that the production of $\mathrm{NO}$ following microglial activation causes a decline in cellular GSH levels leading to brain oxidative damage (Moss and Bates, 2001). A study by Heales et al. (1996) examined brain glutathione and nitric oxide synthase activity (which generates NO). They found that loss of GSH was accompanied by a significant increase in brain nitric oxide synthase activity, by up to $55 \%$. Depletion of GSH in cultured neurons, by approximately $90 \%$, led to a significant $67 \%$ increase in nitric oxide synthase activity, as judged by nitrite formation, and cell death. It was concluded by Heales and colleagues that depletion of neuronal GSH results in increased nitric oxide synthase activity.

\section{EVIDENCE DF INCREASED NITRIC OXIDE PRDDUCTION AND RELATED MEDICAL SYMPTOMSIN ASD}

As mentioned earlier, once activated, microglia release large amounts of NO and superoxide as a cytotoxic attack mechanism (Colton and Gilbert, 1987). ROS and RNS derived from $\mathrm{NO}$ and superoxide may also cause local cellular damage by reacting with proteins, lipids and nucleic acids (Valko et al., 2007). These chemicals can directly damage cells and lead to neuronal cell death. As a result, elevated NO levels can cause a wide array of medical problems, many of which are found in ASD. Although an ASD diagnosis is defined by three core features (impairment in communication and socialization, and behavioral issues), other features, more physical or systemic in nature, are associated with an ASD diagnosis.

For example, a recent analysis of the National Health Interview Survey, 2006-2010, that included 375 children with autism by Schieve et al. (2011), found that children with autism were more likely to have headaches/migraines, respiratory and food allergies, physician visits and to be taking prescription medication than children without autism. Children with ASD are also, according to a study by Atladóttir et al. (2010), more likely to be hospitalized for an infectious disease. These medical diseases could possibly be a result of, or associated with, or exacerbated by elevated NO levels. Some examples are as follows:

As mentioned, children with ASD have a higher rate of infection (Atladóttir et al., 2010). There is evidence that abundant $\mathrm{NO}$ at an inflammatory site may reduce and impair natural killer (NK) cell function (Takabayashi et al., 2000) which may provide an explanation for the frequent infections that a large subset of children with autism suffer from (Nicolson et al., 2007). Studies have found low NK function in ASD (Enstrom et al., 2009). Vojdani et al. (2008), for example, found that at least $45 \%$ of children with autism suffer from low NK cell activity.

Seizures are common in autism, occurring in $20-30 \%$ of patients based on the majority of studies. Epileptiform EEG abnormalities are present in $10.3-72.4 \%$ of patients (Danielsson et al., 2005; Kagan-Kushnir et al., 2005). Several studies have found that microglial activation can result in seizures (Radewicz et al., 2000; Somera-Molina et al., 2007, 2009). In a study by Kovács et al. (2009) the researchers propose that NO-dependent enhancement of synaptic transmission is a key promoting factor for the initiation of seizures. In addition, NO might exert long-term effects in epilepsy. NO-dependent inhibition of mitochondrial electron transport chain activity (Brown, 2001), disruption of the mitochondrial networks (Yuan et al., 2007), and blockade of mitochondrial trafficking (Rintoul et al., 2006) might contribute to metabolic impairment as described for the epileptic hippocampus (Kunz et al., 2000; Kann et al., 2005).

In the National Health Interview Survey, 2006-2010, Schieve et al. (2011) also reported a higher rate of asthma and bronchitis in children with intellectual disabilities (ID), including ASD. In a review by Ashutosh (2000), it was reported that an increase in the exhaled NO has been shown to accompany eosinophilic inflammation and to correlate with other indices of inflammation in asthma. Exhaled NO increases during exacerbation and decreases with recovery in patients with asthma. Yates (2001) also reported that asthma is characterized by chronic airway inflammation and increased synthesis of NO and other highly reactive and toxic substances (ROS). Pro-inflammatory cytokines such as TNF- $\alpha$ and IL- $1 \beta$ are secreted in asthma and result in inflammatory cell recruitment, but also induce calcium- and calmodulin-iNOS and perpetuate the inflammatory response within the airways. NO is released by several pulmonary cells including epithelial cells, eosinophils and macrophages, and NO has been shown to be increased in conditions associated with airway inflammation, such as asthma and viral infections.

Research suggests relatively high rates of eczema and food allergies in ASD as compared to TD children (Schieve et al., 2011). It has been suggested that NO is an important player in eczema, food allergies, and intestinal inflammation. Eczema is characterized by inflammation of the skin and is commonly associated with food allergy. The results of a study, by Devenney et al. (2010), were able to support previous studies indicating that the homeostasis of nitrogen radicals is disturbed in childhood eczema. 


\section{Microglial Activation in Autism}

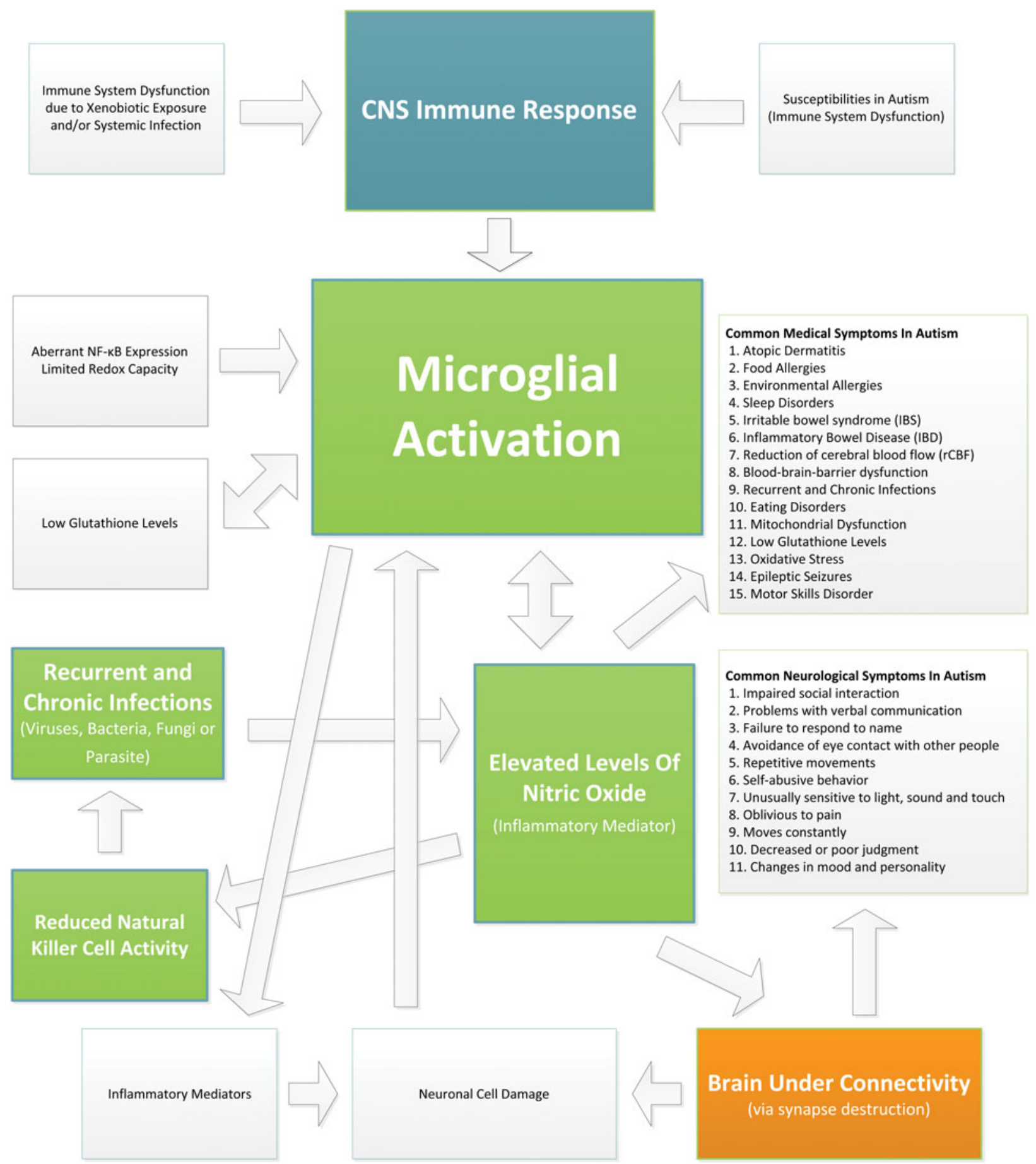

Fig. 1. This diagram shows the relationships and interplay between microglial activation and the neuropathology, medical issues and symptoms in ASD.

As mentioned, Adams et al. (2011) and Wang et al. (2011) found that there is a correlation of gastrointestinal symptoms with autism severity indicating that children with more severe autism are likely to have more severe gastrointestinal symptoms and vice versa. Schieve et al. (2011) found that children with autism were $70 \%$ more likely than children in the ID group, two times more likely than children in the attention- deficit hyperactivity disorder and learning disabled/other developmental delay groups, and seven times more likely than children without developmental delays (DDs) to have had frequent diarrhea/colitis in the last 12 months. Research shows that exaggerated or uncontrolled expression of iNOS itself becomes detrimental to the gastrointestinal tract (Calatayud et al., 2001), and that large amounts of NO can 
increase gut permeability and induce apoptosis (Dijkstra et al., 2004). Inflammatory bowel disease (IBD) and irritable bowel syndrome (IBS) are chronic diseases that cause inflammation of the intestines. A study by Reinders et al. (2005) found that NO was low in healthy control subjects, and variations over time were small. In IBS patients NO was slightly elevated, whereas patients with active IBD or collagenous colitis had greatly increased NO levels. Rectal NO correlated with disease activity in IBD and collagenous colitis and decreased markedly in IBD patients responding to anti-inflammatory treatment.

A statistically significant global reduction of cerebral blood flow (CBF) is found in autistic children (Burroni et al., 2008). Recent studies on brain circulation have provided evidence that $\mathrm{CBF}$ is impaired by decreased formation of NO from endothelial cells, autonomic nitrergic nerves or brain neurons and also by increased production of ROS. The NOROS interaction is an important topic in discussing blood flow and cell viability in the brain (Toda et al., 2009).

In the recent study, Giulivi et al. (2010) found that children with autism were more likely to have mitochondrial dysfunction than TD children. Evidence has also been provided that mitochondrial dysfunction can be induced by elevated levels of NO (Stewart and Heales, 2003). NO and its toxic metabolite peroxynitrite $(\mathrm{ONOO}(-))$ can inhibit the mitochondrial respiratory chain, leading to energy failure and ultimately cell death. ROS and RNS derived from NO and superoxide may also inhibit mitochondrial brain energy metabolism (Valko et al., 2007), preventing the production of adenosine triphosphate (Bolaños et al., 1995).

Abnormal eating patterns and eating disorders are associated with ASD (Maenner et al., 2011; Tang et al., 2011). A team of researchers in Italy provided evidence on the possible actions of NO on the etiology of eating disorders (Vannacci et al., 2006). In this study, plasma nitrite and cyclic guanosine monophosphate levels were significantly higher in eating disorder patients than in healthy controls.

\section{CONCLUSION}

Evidence indicates that children with ASD suffer from an ongoing neuroinflammatory process in different regions of the brain involving microglial activation. The microglial activation appears to play a role in the brain underconnectivity and other issues in ASD (see Fig. 1 which shows the relationships and interplay between microglial activation and the neuropathology, medical issues and symptoms in ASD). Current therapies typically used in ASD do not directly address the underlying neuroinflammation. It is possible that by reducing brain inflammation and microglial activation, the neurodestructive effects of chronic inflammation could be reduced and allow for neuronal reconnection. Reducing brain inflammation could allow for an improved response to early behavioral and learning intervention measures to be more effective, and ultimately enhance developmental outcomes. Many studies suggest that there are pharmaceutical and nutraceutical treatments that can reduce microglial activation and/or their associated inflammatory cytokines. However, the studies that have examined these potential therapies have not been conducted in ASD. Future studies that examine treatments that may reduce microglial activation and neuroinflammation, and ultimately help to mitigate symptoms in ASD, are warranted.

\section{Statement of interest}

None.

\section{REFERENCES}

Adams J.B., Johansen L.J., Powell L.D., Quig D. and Rubin R.A. (2011) Gastrointestinal flora and gastrointestinal status in children with autism - comparisons to typical children and correlation with autism severity. BMC Gastroenterology 11, 22.

Ahlsen G., Rosengren L., Belfrage M., Palm A., Haglid K., Hamberger A. et al. (1993) Glial fibrillary acidic protein in the cerebrospinal fluid of children with autism and other neuropsychiatric disorders. Biological Psychiatry 33, 734-743.

Al-Yafee Y.A., Al-Ayadhi L.Y., Haq S.H. and El-Ansary A.K. (2011) Novel metabolic biomarkers related to sulfur-dependent detoxification pathways in autistic patients of Saudi Arabia. BMC Neurology 11, 139.

Ashutosh K. (2000) Nitric oxide and asthma: a review. Current Opinion in Pulmonary Medicine 6, 21-25.

Atladóttir H.O., Thorsen P., Schendel D.E., Østergaard L., Lemcke S. and Parner E.T. (2010) Association of hospitalization for infection in childhood with diagnosis of autism spectrum disorders: a Danish cohort study. Archives of Pediatric and Adolescent Medicine 164, 470-477.

Bailey A., Luthert P., Dean A., Harding B., Janota I., Montgomery M. et al. (1998) A clinicopathological study of autism. Brain 121, 889-905.

Barttfeld P., Wicker B., Cukier S., Navarta S., Lew S. and Sigman M. (2011) A big-world network in ASD: dynamical connectivity analysis reflects a deficit in long-range connections and an excess of shortrange connections. Neuropsychologia 49, 254-263.

Belmonte M.K., Allen G., Beckel-Mitchener A., Boulanger L.M., Carper R.A. and Webb S.J. (2004) Autism and abnormal development of brain connectivity. Journal of Neuroscience 24, 9228-9231.

Boger-Megiddo I., Shaw D.W., Friedman S.D., Sparks B.F., Artru A.A., Giedd J.N. et al. (2006) Corpus callosum morphometrics in young children with autism spectrum disorder. Journal of Autism Developmental Disorders 36, 733-739.

Bolaños J.P., Heales S.J., Land J.M. and Clark J.B. (1995) Effect of peroxynitrite on the mitochondrial respiratory chain: differential susceptibility of neurones and astrocytes in primary culture. Journal of Neurochemistry 64, 1965-1972.

Brown G.C. (2001) Regulation of mitochondrial respiration by nitric oxide inhibition of cytochrome c oxidase. Biochimica et Biophysica Acta 1504, 46-57.

Burroni L., Orsi A., Monti L., Hayek Y., Rocchi R. and Vattimo A.G. (2008) Regional cerebral blood flow in childhood autism: a SPET study with SPM evaluation. Nuclear Medicine Communications 29, $150-156$.

Calatayud S., Barrachina D. and Esplugues J.V. (2001) Nitric oxide: relation to integrity, injury, and healing of the gastric mucosa. Microscopy Research and Technique 53, 325-335.

Chatterjee S., Noack H., Possel H. and Wolf G. (2000) Induction of nitric oxide synthesis lowers intracellular glutathione in microglia of primary glial cultures. Glia 29, 98-101.

Chauhan A., Audhya T. and Chauhan V. (2012) Brain region-specific glutathione redox imbalance in autism. Neurochemical Research (Epub ahead of print).

Chez M.G., Dowling T., Patel P.B., Khanna P. and Kominsky M. (2007) Elevation of tumor necrosis factor-alpha in cerebrospinal fluid of autistic children. Pediatric Neurology 36, 361-365. 
Colton C.A. and Gilbert D.L. (1987) Production of superoxide anions by a CNS macrophage, the microglia. FEBS Letters 223, 284-288.

Courchesne E. (1997) Brainstem, cerebellar and limbic neuroanatomical abnormalities in autism. Current Opinion in Neurobiology 7, 269-278.

Courchesne E. and Pierce K. (2002) Autism. In Ramachandran V.S. (ed) Encyclopedia of the Human Brain. Academic, San Diego, pp. 321-342.

Damarla S.R., Keller T.A., Kana R.K., Cherkassky V.L., Williams D.L., Minshew N.J. et al. (2010) Cortical underconnectivity coupled with preserved visuospatial cognition in autism: evidence from an fMRI study of an embedded figures task. Autism Research 3, 273-279.

Danielsson S., Gillberg I.C., Billstedt E., Gillberg C. and Olsson I. (2005) Epilepsy in young adults with autism: a prospective populationbased follow-up study of 120 individuals diagnosed in childhood. Epilepsia 46, 918-923.

Devenney I., Norrman G., Forslund T., Fälth-Magnusson K. and Sundqvist T. (2010) Urinary nitric oxide excretion in infants with eczema. Pediatric Allergy and Immunology 21, e229-e234.

Dijkstra G., van Goor H., Jansen P.L. and Moshage H. (2004) Targeting nitric oxide in the gastrointestinal tract. Current Opinion in Investigational Drugs 5, 529-536.

Di Martino A., Kelly C., Grzadzinski R., Zuo X.N., Mennes M., Mairena M.A. et al. (2011) Aberrant striatal functional connectivity in children with autism. Biological Psychiatry 69, 847-856.

Ebisch S.J., Gallese V., Willems R.M., Mantini D., Groen W.B., Romani G.L. et al. (2011) Altered intrinsic functional connectivity of anterior and posterior insula regions in high-functioning participants with autism spectrum disorder. Human Brain Mapping 32, 1013-1028.

Enstrom A.M., Lit L., Onore C.E., Gregg J.P., Hansen R.L., Pessah I.N. et al. (2005) Immunity, neuroglia and neuroinflammation in autism. International Review of Psychiatry 17, 485-495.

Enstrom A.M., Lit L., Onore C.E., Gregg J.P., Hansen R.L., Pessah I.N. et al. (2009) Altered gene expression and function of peripheral blood natural killer cells in children with autism. Brain Behavior and Immunity 23, 124-133.

Fatemi S.H., Folsom T.D., Reutiman T.J. and Lee S. (2008) Expression of astrocytic markers aquaporin 4 and connexin 43 is altered in brains of subjects with autism. Synapse 62, 501-507.

Gehrmann J., Matsumoto Y. and Kreutzberg G.W. (1995) Microglia: intrinsic immuneffector cell of the brain. Brain Research Reviews 20, 269-287.

Geier D.A., Kern J.K., Garver C.R., Adams J.B., Audhya T. and Geier M.R. (2009) A prospective study of transsulfuration biomarkers in autistic disorders. Neurochemical Research 34, 386-393.

Giulivi C., Zhang Y.F., Omanska-Klusek A., Ross-Inta C., Wong S., Hertz-Picciotto I. et al. (2010) Mitochondrial dysfunction in autism. Journal of the American Medical Association 304, 2389-2396.

Heales S.J., Bolaños J.P. and Clark J.B. (1996) Glutathione depletion is accompanied by increased neuronal nitric oxide synthase activity. Neurochemical Research 21, 35-39.

Herbert M.R. (2005) Large brains in autism: the challenge of pervasive abnormality. Neuroscientist 11, 417-440.

Herbert M.R., Ziegler D.A., Deutsch C.K., O’Brien L.M., Kennedy D.N., Filipek P.A. et al. (2005) Brain asymmetries in autism and developmental language disorder: a nested whole-brain analysis. Brain 128, 213-226.

Herbert M.R., Ziegler D.A., Makris N., Filipek P.A., Kemper T.L., Normandin J.J. et al. (2004) Localization of white matter volume increase in autism and developmental language disorder. Annals of Neurology 55, 530-540.
Hirrlinger J., Gutterer J.M., Kussmaul L., Hamprecht B. and Dringen R. (2000) Microglial cells in culture express a prominent glutathione system for the defense against reactive oxygen species. Developmental Neuroscience 22, 384-392.

James S.J., Cutler P., Melnyk S., Jernigan S., Janak L., Gaylor D.W. et al. (2004) Metabolic biomarkers of oxidative stress and impaired methylation capacity in children with autism. American Journal of Clinical Nutrition 80, 1611-1617.

James S.J., Melnyk S., Jernigan S., Cleves M.A., Halsted C.H., Wong D.H. et al. (2006) Metabolic endophenotype and related genotypes are associated with oxidative stress in children with autism. American Journal of Medical Genetics Part B, Neuropsychiatric Genetics 141B, 947-956.

James S.J., Rose S., Melnyk S., Jernigan S., Blossom S., Pavliv O. et al. (2009) Cellular and mitochondrial glutathione redox imbalance in lymphoblastoid cells derived from children with autism. FASEB Journal 23, 2374-2383

Just M.A., Cherkassky V.L., Keller T.A., Kana R.K. and Minshew N.J. (2007) Functional and anatomical cortical underconnectivity in autism: evidence from an FMRI study of an executive function task and corpus callosum morphometry. Cerebellar Cortex 17, 951-961.

Kagan-Kushnir T., Roberts S.W. and Snead O.C. III (2005) Screening electroencephalograms in autism spectrum disorders: evidence-based guideline. Journal of Child Neurology 20, 197-206.

Kana R.K., Keller T.A. and Cherkassky V.L. (2009) Atypical frontalposterior synchronization of Theory of Mind regions in autism during mental state attribution. Society Neuroscience 4, 135-152.

Kann O., Kovács R., Njunting M., Behrens C.J., Otáhal J., Lehmann T.N. et al. (2005) Metabolic dysfunction during neuronal activation in the ex vivo hippocampus from chronic epileptic rats and humans. Brain 128, 2396-2407.

Kigerl K.A., Ankeny D.P., Garg S.K., Wei P., Guan Z., Lai W. et al. (2011) System $\mathrm{x}(\mathrm{c})(-)$ regulates microglia and macrophage glutamate excitotoxicity in vivo. Experimental Neurology 233, 333-341.

Kovács R., Rabanus A., Otáhal J., Patzak A., Kardos J., Albus K. et al. (2009) Endogenous nitric oxide is a key promoting factor for initiation of seizure-like events in hippocampal and entorhinal cortex slices. Journal of Neuroscience 29, 8565-8577.

Kunz W.S., Kudin A.P., Vielhaber S., Blümcke I., Zuschratter W., Schramm J. et al. (2000) Mitochondrial complex I deficiency in the epileptic focus of patients with temporal lobe epilepsy. Annals of Neurology 48, 766-773.

Laurence J.A. and Fatemi S.H. (2005) Glial fibrillary acidic protein is elevated in superior frontal, parietal and cerebellar cortices of autistic subjects. Cerebellum 4, 206-210.

Lee R.H., Mills E.A., Schwartz N., Bell M.R., Deeg K.E., Ruthazer E.S. et al. (2010) Neurodevelopmental effects of chronic exposure to elevated levels of pro-inflammatory cytokines in a developing visual system. Neural Development 5, 2.

Li X., Chauhan A., Sheikh A.M., Patil S., Chauhan V., Li X.M. et al. (2009) Elevated immune response in the brain of autistic patients. Journal of Neuroimmunology 207, 111-116.

Maenner M.J., Arneson C.L., Levy S.E., Kirby R.S., Nicholas J.S. and Durkin M.S. (2011) Brief report: association between behavioral features and gastrointestinal problems among children with autism spectrum disorder. Journal of Autism and Developmental Disorders [Epub ahead of print]

Minshew N.J. and Keller T.A. (2010) The nature of brain dysfunction in autism: functional brain imaging studies. Current Opinion in Neurology 23, 124-130. 
Monnet-Tschudi F., Defaux A., Braissant O., Cagnon L. and Zurich M.G. (2011) Methods to assess neuroinflammation. Current Protocols in Toxicology, Chapter 12, Unit 12.19.

Morgan J.T., Chana G., Pardo C.A., Achim C., Semendeferi K., Buckwalter J. et al. (2010) Microglial activation and increased microglial density observed in the dorsolateral prefrontal cortex in autism. Biological Psychiatry 68, 368-376.

Moss D.W. and Bates T.E. (2001) Activation of murine microglial cell lines by lipopolysaccharide and interferon- $\gamma$ causes NO-mediated decreases in mitochondrial and cellular function. European Journal of Neuroscience 13, 529-538.

Naik U.S., Gangadharan C., Abbagani K., Nagalla B., Dasari N. and Manna S.K. (2011) A study of nuclear transcription factor-kappa B in childhood autism. PLoS One 6, e19488.

Nakanishi H., Hayashi Y. and Wu Z. (2011) The role of microglial mtDNA damage in age-dependent prolonged LPS-induced sickness behavior. Neuron Glia Biology 28, 1-7.

Nicolson G.L., Gan R., Nicolson N.L. and Haier J. (2007) Evidence for Mycoplasma ssp., Chlamydia pneunomiae, and human herpes virus- 6 coinfections in the blood of patients with autistic spectrum disorders. Journal of Neuroscience Research 85, 1143-1148.

Palmen S.J., van Engeland H., Hof P.R. and Schmitz C. (2004) Neuropathological findings in autism. Brain 127, 2572-2583.

Pardo C.A., Vargas D.L. and Zimmerman A.W. (2005) Immunity, neuroglia and neuroinflammation in autism. International Review of Psychiatry 17, 485-495.

Radewicz K., Garey L.J., Gentleman S.M. and Reynolds R. (2000) Increase in HLA-DR immunoreactive microglia in frontal and temporal cortex of chronic schizophrenics. Journal of Neuropathology and Experimental Neurology 59, 137-150.

Reinders C.I., Herulf M., Ljung T., Hollenberg J., Weitzberg E., Lundberg J.O. et al. (2005) Rectal mucosal nitric oxide in differentiation of inflammatory bowel disease and irritable bowel syndrome. Clinical Gastroenterology and Hepatology 3, 777-783.

Rintoul G.L., Bennett V.J., Papaconstandinou N.A. and Reynolds I.J. (2006) Nitric oxide inhibits mitochondrial movement in forebrain neurons associated with disruption of mitochondrial membrane potential. Journal of Neurochemistry 97, 800-806.

Rosengren L.E., Ahlsén G., Belfrage M., Gillberg C., Haglid K.G. and Hamberger A. (1992) A sensitive ELISA for glial fibrillary acidic protein: application in CSF of children. Journal of Neuroscience Methods 44, 113-119.

Schieve L.A., Gonzalez V., Boulet S.L., Visser S.N., Rice C.E., Braun K.V. et al. (2011) Concurrent medical conditions and health care use and needs among children with learning and behavioral developmental disabilities, National Health Interview Survey, 2006-2010. Research in Developmental Disabilities 33, 467-476.

Shukla D.K., Keehn B., Lincoln A.J. and Müller R.A. (2010) White matter compromise of callosal and subcortical fiber tracts in children with autism spectrum disorder: a diffusion tensor imaging study. Journal of the American Academy of Child \& Adolescent Psychiatry 49, 1269-1278.

Somera-Molina K.C., Nair S., Van Eldik L.J., Watterson D.M. and Wainwright M.S. (2009) Enhanced microglial activation and proinflammatory cytokine upregulation are linked to increased susceptibility to seizures and neurologic injury in a 'two-hit' seizure model. Brain Research 1282, 162-172.

Somera-Molina K.C., Robin B., Somera C.A., Anderson C., Stine C., Koh S. et al. (2007) Glial activation links early-life seizures and long-term neurologic dysfunction: evidence using a small molecule inhibitor of proinflammatory cytokine upregulation. Epilepsia 48, 1785-1800.

Stewart V.C. and Heales S.J. (2003) Nitric oxide-induced mitochondrial dysfunction: implications for neurodegeneration. Free Radical Biology and Medicine 34, 287-303.

Streit W.J., Mrak R.E. and Griffin W.S. (2004) Microglia and neuroinflammation: a pathological perspective. Journal of Neuroinflammation 1,14 .

Streita W.J. (2006) Microglial senescence: does the brain's immune system have an expiration date? Trends in Neuroscience 29, 506-510.

Takabayashi A., Kawai Y., Iwata S., Kanai M., Denno R., Kawada K. et al. (2000) Nitric oxide induces a decrease in the mitochondrial membrane potential of peripheral blood lymphocytes, especially in natural killer cells. Antioxidants and Redox Signaling 2, 673-680.

Tang B., Piazza C.C., Dolezal D. and Stein M.T. (2011) Severe feeding disorder and malnutrition in 2 children with autism. Journal of Developmental and Behavioral Pediatrics 32, 264-267.

Tetreault N.A., Hakeem A.Y., Jiang S., Williams B.A., Allman E., Wold B.J. et al. (2012) Microglia in the cerebral cortex in autism. Journal of Autism and Developmental Disorders [Epub ahead of print].

Toda N., Ayajiki K. and Okamura T. (2009) Cerebral blood flow regulation by nitric oxide in neurological disorders. Canadian Journal of Physiology and Pharmacology 87, 581-594.

Valko M., Leibfritz D., Moncol J., Cronin M.T., Mazur M. and Telser J. (2007) Free radicals and antioxidants in normal physiological functions and human disease. International Journal of Biochemistry and Cell Biology 39, 44-84.

Vannacci A., Ravaldi C., Giannini L., Rotella C.M., Masini E., Faravelli C. et al. (2006) Increased nitric oxide production in eating disorders. Neuroscience Letters 399, 230-233.

Vargas D.L., Nascimbene C., Krishnan C., Zimmerman A.W. and Pardo C.A. (2005) Neuroglial activation and neuroinflammation in the brain of patients with autism. Annals of Neurology 57, 304 .

Vojdani A., Mumper E., Granpeesheh D., Mielke L., Traver D., Bock K. et al. (2008) Low natural killer cell cytotoxic activity in autism: the role of glutathione, IL-2 and IL-15. Journal of Neuroimmunology 205, 148154 .

Wang L.W., Tancredi D.J. and Thomas D.W. (2011) The prevalence of gastrointestinal problems in children across the United States with autism spectrum disorders from families with multiple affected members. Journal of Developmental and Behavioral Pediatrics 32, 351-360.

Wass S. (2011) Distortions and disconnections: disrupted brain connectivity in autism. Brain Cognition 75, 18-28.

Wood P.L. (2003) Roles of CNS macrophages in neurodegeneration. In Neuroinflammation. Mechanisms and Management, 2nd edition. P.L. Wood (ed.). Humana Press, Totowa, NJ, pp. 3-27.

Yates D.H. (2001) Role of exhaled nitric oxide in asthma. Immunology and Cell Biology 79, 178-190.

Young A.M., Campbell E., Lynch S., Suckling J. and Powis S.J. (2011) Aberrant NF-kappaB expression in autism spectrum condition: a mechanism for neuroinflammation. Frontiers in Psychiatry 2, 27.

Yuan H., Gerencser A.A., Liot G., Lipton S.A., Ellisman M., Perkins G.A. et al. (2007) Mitochondrial fission is an upstream and required event for bax foci formation in response to nitric oxide in cortical neurons. Cell Death and Differentiation 14, 462-471.

Zimmerman A.W., Jyonouchi H., Comi A.M., Connors S.L., Milstein S., Varsou A. et al. (2005) Cerebrospinal fluid and serum markers of inflammation in autism. Pediatric Neurology 33, 195-201. 


\section{AUTHORS'ADDRESSES}

1 Stop Calling It Autism, Fort Worth, TX, USA

2 Institute of Chronic Illnesses Inc., Allen, TX, USA

3 University of Texas Southwestern Medical Center, Dallas, TX, USA
Correspondence should be addressed to:

Janet K. Kern

Institute of Chronic Illnesses Inc.

408 North Allen Drive

Allen, TX 75013

USA

phone: (214)592-6600

fax: 1 (888)SCIA-123 or $1(888) 724-2123$

email: jkern@dfwair.net 